\title{
Evaluation of Electrical Resistivity of Oxide Scale Formed under Different Conditions*
}

\author{
Md. Rostom ALI**, Masumi SAKA**, Hironori TOHMYOH** \\ and M. A. Salam AKANDA** \\ **Department of Nanomechanics, Tohoku University \\ Aoba 6-6-01, Aramaki, Aoba-ku, Sendai 980-8579, Japan \\ (Corresponding author E-mail: mrali@ism.mech.tohoku.ac.jp)
}

\begin{abstract}
The nature of direct current potential drop (DCPD) on carbon steel (SS400) having different surface conditions is investigated in this study. The spring-loaded macroscopic DC four-point-probe sensor was used for the measurements of potential drop. Three samples from the same type of carbon steel are prepared under three different conditions, namely, exposures for short and long periods in atmosphere and heat treatment at low temperature of the sample experienced short exposure. In an attempt to verify the repeatability nature of DCPD, the measurements are performed on different locations of the test surface under the same experimental conditions and used for analysis. The natures of potential drop on oxide scale free surface and oxidized surfaces were compared. The effect of oxidized surfaces on DCPD in all the three cases is observed markedly. From these observations, it is worth understanding that the DCPD technique through spring-loaded macroscopic four-point-probe is able to distinguish the oxidized surface from oxide scale free surface. Finally, electrical resistivities of oxide scale in all the three cases were successfully evaluated by using electrical image method with oxidized sample considering as a two-layer structure. The almost unique value of evaluated electrical resistivity of oxide scale in all the cases indicates that the oxide materials are similar in type in respect to electrical resistivity.
\end{abstract}

Key words: Electrical Resistivity, Oxide Scale, DCPD, Electrical Image Method, Two-Layer

\section{Introduction}

DC electrical resistivity measurement is a powerful tool in the investigation of many solid state phenomena ${ }^{(1,2)}$. Experimentally electrical conductivity or resistivity has been studied very intensively for about a hundred years for understanding of many aspects of electrical conduction and properties of conductor in general ${ }^{(3)}$. The importance of ensuring the metal surface cleanliness/checking is necessary before plating, painting or any coating operation for quality adhesion and requiring maintenance free protection of any structural materials. Therefore metal surface should be checked before coating operation to avoid poor adhesion. The interaction of coatings with metallic or oxide surfaces has been an important issue for many years in all kinds of research fields, like corrosion science, catalysis, and optoelectronics $^{(4)}$. In corrosive environments, corrosion protective organic coating on steel surface will often fail due to delamination of coating. The electric resistance of oxide scale on the steel surface is one of the crucial parameters to the delamination rate. Therefore knowing of the electrical resistivity of oxide layer is important for the corrosion protection system $^{(5)}$. 
During the process of hot rolling and subsequent of cooling, oxide scale forms on the surface of the hot-rolled strip steels ${ }^{(6,7)}$. A number of different factors in hot rolling process are considered for energy requirements to produce quality products ${ }^{(8)}$. The electrical resistivity of the oxide layer is one of the factors in the research of hot rolling process ${ }^{(9)}$. Oxidation improves the magnetic properties of hot-rolled electrical steels like transformer steels ${ }^{(10)}$. For optimization of magnetic properties, investigation of electrical resistivity of the oxide layer is also important. Therefore, the evaluation of the electrical resistivity of the oxide scales formed on steels under different conditions of temperature, atmosphere and duration is essentially important.

The spring-loaded macroscopic DC potential drop measuring system was developed for determination of electrical resistivity of deposited oxide layer on large base metal ${ }^{(11)}$. The measurement system usually shows the scattering nature of the potential drop data measured on different locations of the surface of an inspected material under the same experimental condition. This scattering is due to the electrical noise, the contact resistance between the probe tip and the test surface, and eccentricity of the probe tip for the clearances between probe and probe guide ${ }^{(12)}$. In this investigation, using a large number of measurements the scatter band is determined and the technique is used for the evaluation of electrical resistivities of the oxide scales formed on steels under different conditions.

In the present approach, to establish the applicability of the technique to different conditions of scale materials, three samples from same carbon steel are prepared under three different conditions. Pre-painted manufactured steels are normally exposed to atmosphere in different durations before supplied to appliances industry. The sample conditions are also selected to imitate these steels for studying the resistivity of their oxide scale. Depending on the condition of sample preparation, thickness and/or mechanical properties of oxide scale may differ sample to sample. The electrical resistivities of oxide scale in all the three cases were successfully evaluated from DCPD measurements and compared. The present paper established that the DCPD technique for evaluation of electrical resistivity of oxide scale is independent of PD decreasing nature with probe contact time. This study also described the decreasing nature of PD as a result of the indentation of oxide layer with probe contact time.

\section{Experimental Details}

In this experiment same type of carbon steel (SS400) samples with three different conditions, namely, exposure for a short period to atmosphere, for a long period to atmosphere and heat treatment were used for measurements of potential drop. The samples for short period exposure, long period exposure and heat treated are designated in this article by S-1, S-2, and S-3, respectively. The machined sample exposed to atmosphere for approximately three months is considered as a sample experienced a short exposure (S-1) and the sample exposed to atmosphere after machining for approximately three years is considered as a sample experienced a long exposure (S-2). These samples were exposed to indoor atmosphere to ensure formation of a stable oxide layer on the sample surface. The sample with short exposure was heated at $324.6 \mathrm{~K}$ for four hours by applying hot air stream using a hair dryer and the sample is termed here as heat treated (S-3). The surface temperature of the heat treated sample was measured by K-type thermocouple. Oxide layer properties of heat treated sample are expected to be changed from that of the sample with short exposure due to forced oxidation by heating at low temperature with air stream.

In this study, a large sensor block is used to measure potential drop having basement size of $210 \mathrm{~mm} \times 110 \mathrm{~mm}$. Therefore, the compatible size of the test sample necessarily becomes very big. The minimum size of inspected samples was $300 \mathrm{~mm} \times 300 \mathrm{~mm} \times 40 \mathrm{~mm}$. At first the potential drops on the surface of the sample with oxide layer were measured by means of DC four-point-probe PD method. Then the flat surface of the sample was rubbed 
with series of abrasive emery papers up to the mesh number 1600 (180-400-500-700-10001500-1600 grit) to remove the oxide layer. The potential drops on the oxide scale free samples were also measured with the same experimental conditions.

The simplified experimental arrangement of DCPD measuring system is shown in Fig. 1. The spring assembly probes were brought into contact with test sample by accurate movement of an actuator ${ }^{(12)}$. A constant $15 \mathrm{~A}$ direct current was applied through outer probes and potential drop was measured between inner probes. The current supply and measurement of potential drop were provided through a simple compression spring-loaded contact probes. The force of spring pushing a current probe to a test surface was $8.31 \mathrm{~N}$ and diameter of the probe tip was $0.75 \mathrm{~mm}^{(11)}$. Initially switch $1\left(\mathrm{SW}_{1}\right)$ is connected and switch $2\left(\mathrm{SW}_{2}\right)$ is kept off for a period of 50-60 minutes to ensure the stability of current supply. $\mathrm{SW}_{2}$ is connected during the measurement whereas $\mathrm{SW}_{1}$ is kept off. A shunt resistor of accuracy $\pm 0.05 \%$ with a digital multimeter is incorporated in the system to monitor the supplied current during the measurement. Measurements of potential drop were carried out at the seven different locations on the same surface of test sample. A total of 10 potential drops data were recorded in one contact of probe to the sample surface in 15 seconds at the same location. Therefore, the data acquisition interval was 1.5 seconds. All measurements of potential drop were carried out at room temperature.

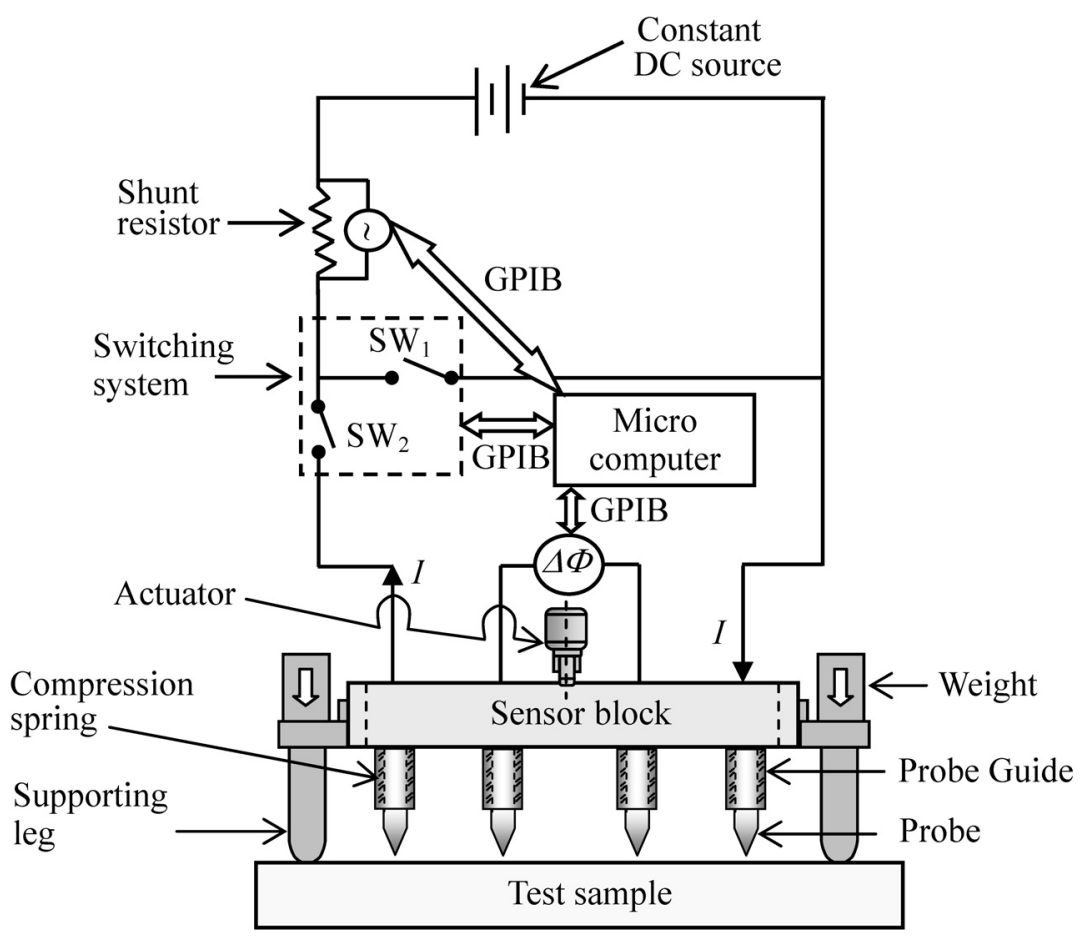

Fig. 1 Schematic of the simplified experimental arrangement of DCPD measuring system.

\section{Theory of the Method}

When metallic surfaces are exposed to atmosphere, they gradually change to oxidized surfaces with time. The electrical potential drop measurement for a metal surface is affected by any foreign material deposited on the surface. In case of oxidized test sample, the base material having large thickness is covered with thin oxide layer of thickness $t$. In this paper, oxidized and oxide scale free test samples are considered as a two-layer and one-layer 
structures, respectively. For two-layer test sample, the parameters $\rho_{1}$ and $\rho_{2}$ are defined as the electrical resistivities of the top layer, oxide scale, and the bottom layer, base material, respectively, as shown in Fig. 2. The distances of the current probes and the potential drop measuring probes from the center of the sensor block are designated by $S_{1}$ and $S_{2}$, respectively. The expressions for potential drop in inner probes due to the outer current source and drain probes on the two-layer test surface can be obtained by electrical image $\operatorname{method}^{(13)}$. When the tips of all the probes are located on the surface of the top layer, the potential drop between two measuring probes $\Delta \Phi$ for two-layer test sample can be expressed by ${ }^{(13)}$

$$
\begin{aligned}
\Delta \Phi=\Phi_{1}-\Phi_{2} & =\frac{\rho_{1} I}{\pi} \times \frac{1}{S_{1}-S_{2}}\left[1+2 \sum_{m=1}^{\infty} k^{m}\left\{1+4 m^{2}\left(\frac{t}{S_{1}-S_{2}}\right)^{2}\right\}^{-1 / 2}\right] \\
& -\frac{\rho_{1} I}{\pi} \times \frac{1}{S_{1}+S_{2}}\left[1+2 \sum_{m=1}^{\infty} k^{m}\left\{1+4 m^{2}\left(\frac{t}{S_{1}+S_{2}}\right)^{2}\right\}^{-1 / 2}\right],
\end{aligned}
$$

where $k\left[=\left(\rho_{2}-\rho_{1}\right) /\left(\rho_{2}+\rho_{1}\right)\right]$ is a reflection coefficient. In Eq. (1), $k$ takes values which lie between \pm 1 in any cases, depending on the relative resistivities of the two layers; the value of $k$ lies $-1<k<0$ and $0<k<1$ for the cases of $\rho_{1}>\rho_{2}$ and $\rho_{1}<\rho_{2}$, respectively ${ }^{(13)}$. According to the experimental conditions, $t<<\left(S_{1}-S_{2}\right)$ and $t<<\left(S_{1}+S_{2}\right)$; the terms in parenthesis of Eq. (1) are negligible, i.e., $t /\left(S_{1}-S_{2}\right) \approx 0$ and $t /\left(S_{1}+S_{2}\right) \approx 0$. Then Eq. (1) is simplified as ${ }^{(11)}$

$$
\Delta \Phi=\frac{2 \rho_{1} I}{\pi} \times \frac{S_{2}}{S_{1}^{2}-S_{2}^{2}}\left(1+2 \sum_{m=1}^{\infty} k^{m}\right) .
$$

Note that Eq. (2) contains the parameter $\rho_{1}$ but it is free from the thickness term $t$ that means Eq. (2) is independent of $t$ only when $t$ is very small compared to probe spacing. On the other hand, the potential drop for one-layer test sample can be expressed by ${ }^{(14)}$

$$
\Delta \Phi=\frac{2 \rho_{2} I}{\pi} \times \frac{S_{2}}{S_{1}^{2}-S_{2}^{2}} .
$$

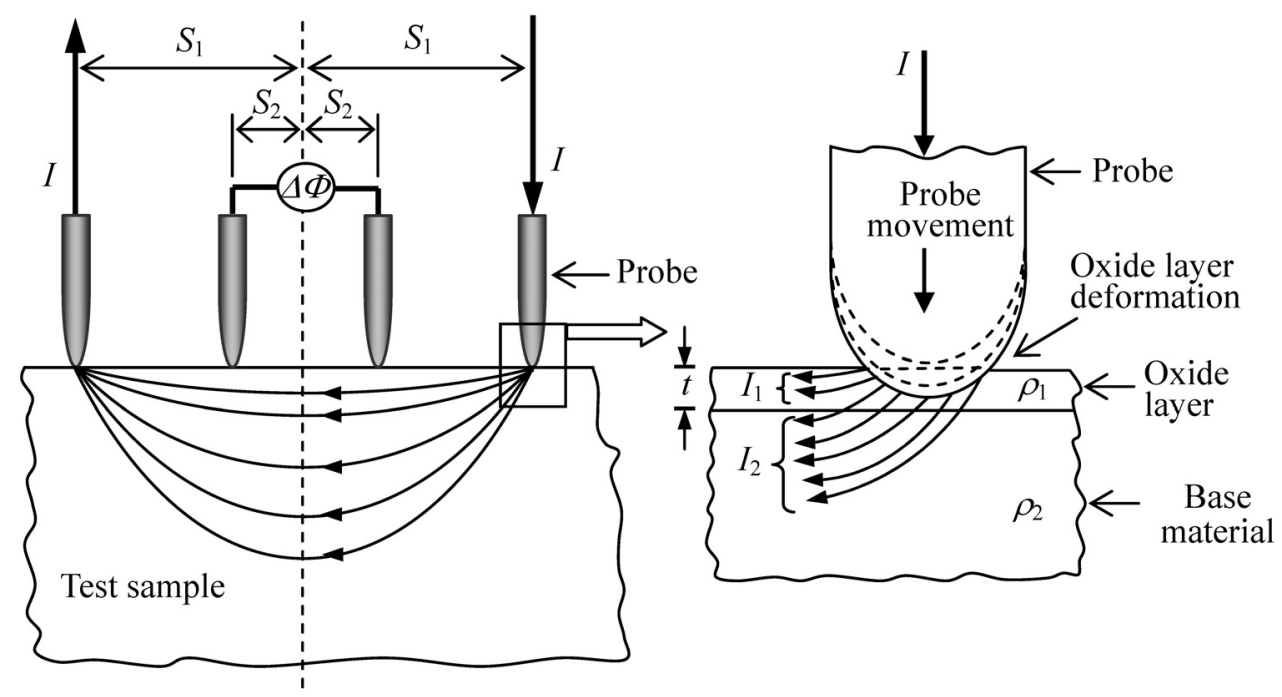

Fig. 2 Definition of symbols and phenomenon of decreasing nature of potential drop. 


\section{Results and Discussions}

\subsection{Nature of Potential Drop on Test Samples}

The potential drops measured at different locations of the test sample were found to be repeatable within a scatter band under the same experimental conditions. For analyzing the sample condition a number of data at different measurement position of the test surface are recorded and their average values together with the standard deviation are used for analysis. The nature of potential drops of the carbon steel (SS400) samples (S-1, S-2, and S-3) is shown in Figs. 3-5. Note that the level as indicated in horizontal axis of Figs. 3-5 is the data acquisition order. The nature of PDs of rubbed surface is also shown in Figs. 3-5. The difference of PD nature with probe contact time for oxidized and rubbed surfaces is observed markedly. Potential drops are decreased on oxidized test surface and remained constant on rubbed surface in each contact of probe.

The surface of carbon steel is covered with the thin oxide layer. During data acquisition Joule heat is generated at the probe contact area continuously with the time of current supply due to contact resistance and the resistance of oxide layer. The Joule heat is highly localized across the tiny contact area and therefore, there occurs rapid overheating ${ }^{(15)}$. The area of contact will no longer be adequate to resist the compressive spring force attached with the probe. Materials across the contact area will go under sharp changes in mechanical, thermal and electrical properties, and all these changes will be localized across the current probe and thus, the material under the probe tip will be deformed gradually with probe contact time due to the spring force as shown in Fig. 2.

As the oxidized test sample is of two layers with the thin oxide layer and the thick base material, a small part of supplied current will flow through the oxide layer. Therefore, the total supplied current $I$ can be divided into $I_{1}$ and $I_{2}$ for the oxide layer and the base material, respectively, as shown in Fig. 2. As observed in Figs. 3-5, the potential drops on oxidized test surface were decreased with probe contact time. This indicates that the current densities in the layers under the measuring probes were changed with probe contact time. The current probe moves towards the surface of more conductive base material due to deformation of the oxide layer under the probe tip as shown in Fig. 2. Due to the deformation, the distance between the probe tip and the base material gradually decreases with probe contact time. Therefore $I_{1}$ decreases and $I_{2}$ increases with the downward movement of the probe. This is the phenomenon of decreasing nature of PD on a two-layer sample.

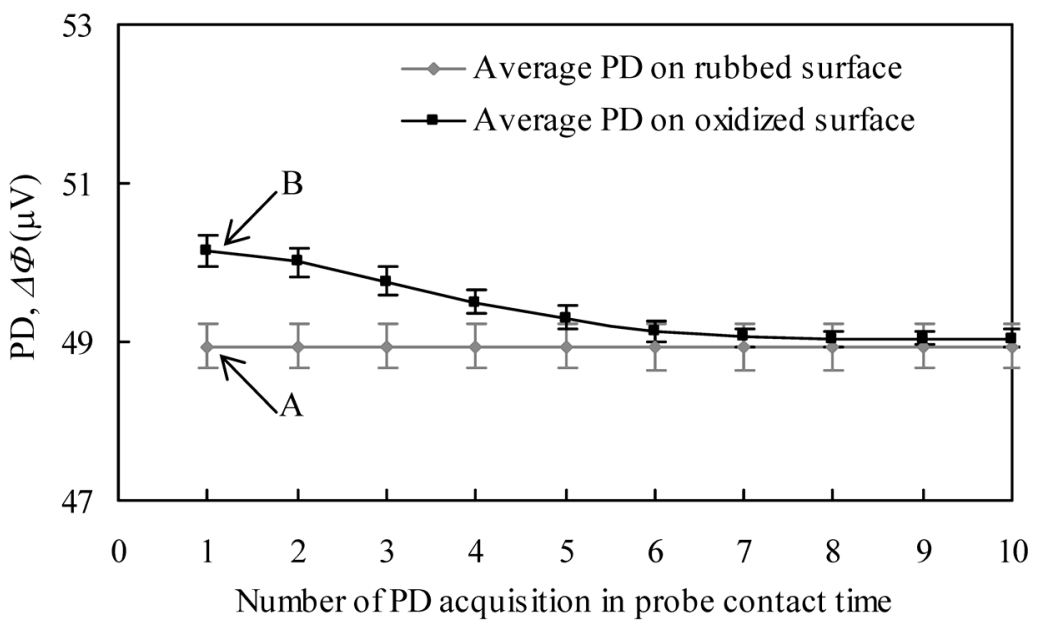

Fig. 3 Nature of PD on short period exposure carbon steel sample (S-1) in atmosphere. 


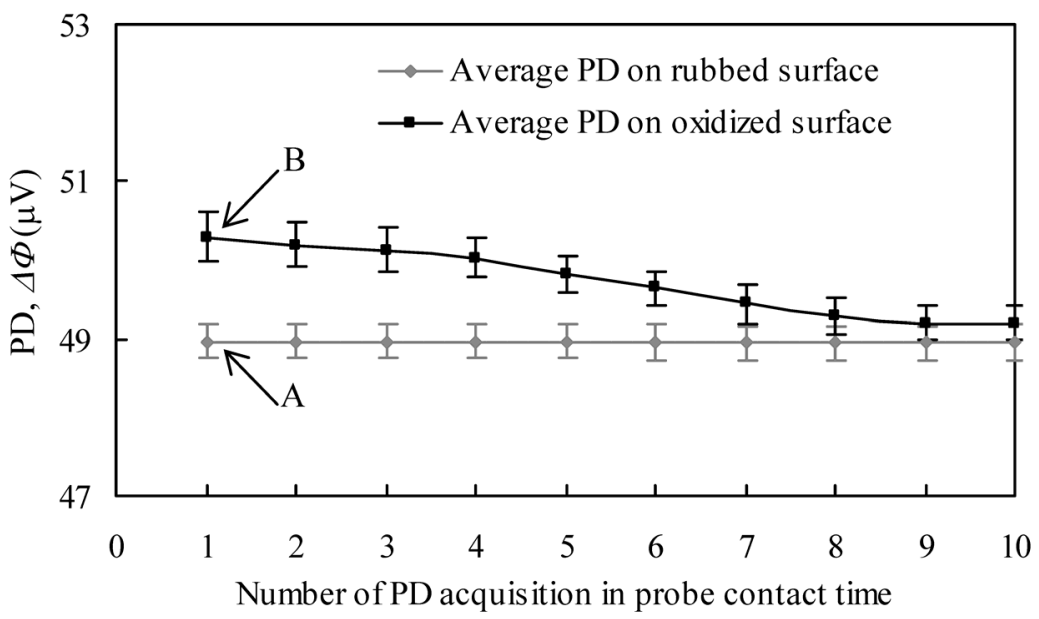

Fig. 4 Nature of PD on long period exposure carbon steel sample (S-2) in atmosphere.

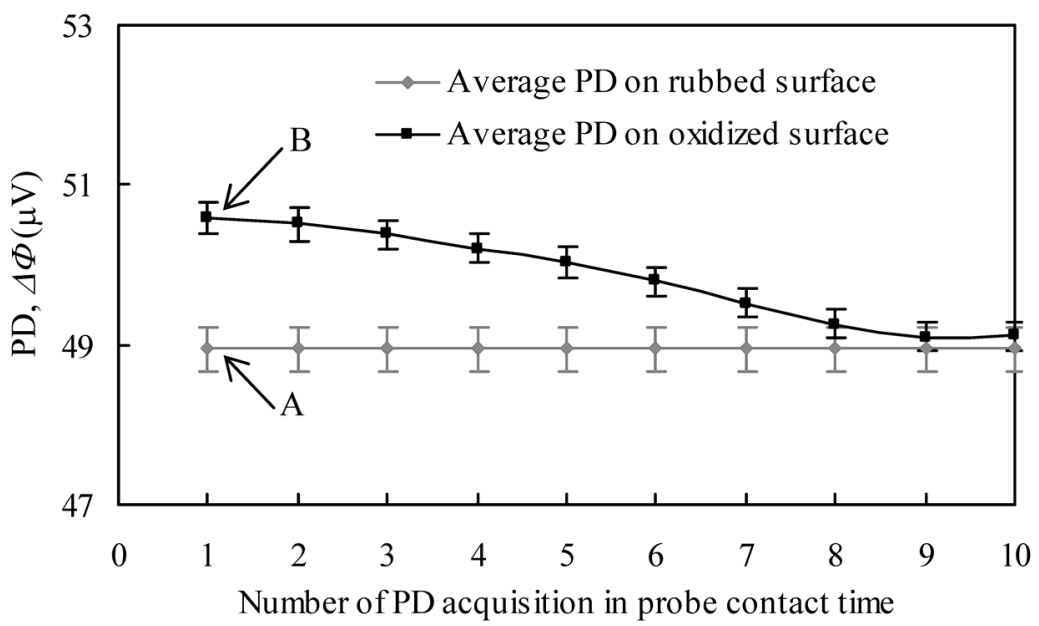

Fig. 5 Nature of PD on heat treated carbon steel sample (S-3).

The PD of oxidized surface decreases until deformation of the oxide scale under the probe tip reaches to an equilibrium state. For the sample with short exposure as observed in Fig. 3, the PD measured on the oxidized surface decreased to PD for the base material at the time of the sixth acquisition. After the sixth acquisition, the PD remains unchanged and shows close concurrent nature with PD for the base material. As observed in Fig. 4, for the sample experienced long exposure, PD measured on the oxidized surface decreased to PD for the base material at the time of ninth acquisition, that means time required to reach equilibrium state is longer. This might be due to the difference in position of the probe tip along the thickness of oxide layer with PD acquisition time. The probe tip position along the thickness of the oxide scale with PD acquisition time depends on thickness and/or mechanical properties of the oxide layer. After the heat treatment of the sample experienced short exposure, the time required to reach the equilibrium state has increased significantly as observed in Fig. 5. This change in nature indicates that the heat treatment process changed the thickness and/or mechanical properties of oxide scale. It is worth understanding that the oxide scale under the probe-tip gradually changes to an equilibrium state due to the high current and contacting pressure, and the time required for this changing depends on the thickness and/or mechanical properties of the scale layer. On the other hand, potential drops 
$(50.2,50.3$, and $50.5 \mu \mathrm{V})$ at the first acquisition in all the three cases are very close to one another. These close values indicate that PD at the first acquisition depends on the resistivities of oxide scale and base material and independent of thickness and/or mechanical properties of oxide scale. The first acquisition of PD data in all the three cases is also free from the surface indentation occurred by the current probes and, therefore, the nature of indentation will not affect the evaluation of electrical resistivity of oxide layer if the first acquisition data is used for evaluation. After the first acquisition, potential drops at later number of acquisitions are affected by thickness and/or mechanical properties of the oxide scale with the base material.

For having a proof of the indentation/deformation of oxide layer that takes place under the current probes during the process of data acquisition, an additional experiment was performed with a condition of scale free contact of current probes with the base material and the results are compared with the results for oxidized and rubbed surfaces as shown in Fig. 6(a). The different measurement conditions are schematically shown in Figs. 6(b d). For obtaining the scale free contact between the current probes and the base material, the oxide scales from the small areas only under the current probes have been removed before the experiment [Fig. 6(c)]. As already described earlier the PD for the scale free base material [Fig. 6(d)] is lowest in magnitude and constant with respect to acquisition time. As observed in Fig. 6(a), the PD for the oxidized surface with the measurement condition as described in Fig. 6(c) is also found constant in magnitude but little higher than that of the base material. This is because although the current probes are in contact with the base material, the inner measuring probes are in contact with the oxide scale. The PD for oxidized surface [Fig. 6(b)] decreases with the acquisition time and becomes steady with the magnitude equal to that obtained for the measurement condition of Fig. 6(c). Therefore, this finding confirms that the current probes gradually moved from the oxide surface to the interface during the acquisition process and finally reached to the base material by the indentation/deformation of the oxide layer.

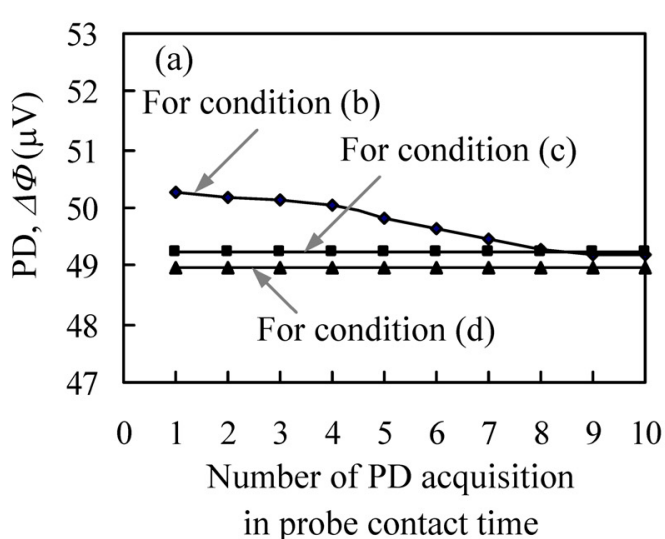

(b)

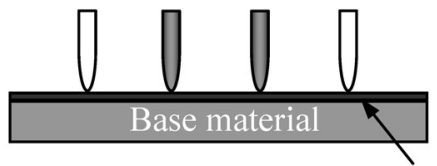

Oxide scale

(c)

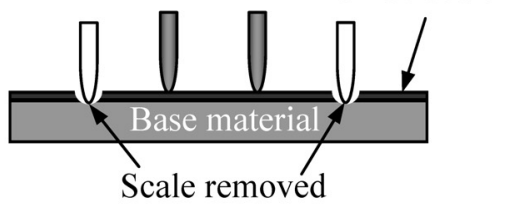

(d)

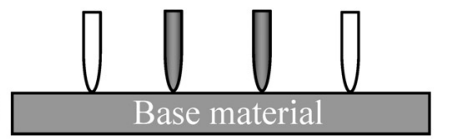

Fig. 6 (a) Nature of PD under different measurement conditions, (b) measurement on oxidized surface, (c) oxide scale under current probes was removed and

(d) measurement on scale free surface.

\subsection{Electrical Resistivity of Oxide Layer}

According to Figs. 3-5, potential drop measured for the oxidized surface gradually decreases with acquisition time and falls to the close value to the potential drop for the base material asymptotically. Therefore, the difference between the potential drops data measured for the oxidized surface and the rubbed surface is highest for the first acquisition. The potential drops at the points A of Figs. 3-5 are influenced only by the resistivity $\rho_{2}$, but 
at the points B they are affected by $\rho_{1}$ and $\rho_{2}$. The PD at position $\mathrm{A}$ is used to determine the electrical resistivity of the base material by Eq. (3). Equation (2) is derived based on the condition that all the probes are located on the surface of the top layer. It can be considered that the PD at the first acquisition in experiment for the oxidized surface satisfies this condition. Therefore, the PD at position $\mathrm{B}$ can be used to determine the electrical resistivity of the oxide scale by Eq. (2). The PDs at any later number of acquisitions are for a state that the current probes are indenting the oxide layer and moving to the base material during the period of contact. Therefore, these data will not consistent with the conditions assumed for deriving Eq. (2) and hence these are not used for the calculation of electrical resistivity. A computer program has been used to determine the value of $\rho_{2} / \rho_{1}$ from Eq. (2). An example of the graphical result is shown in Fig. 7. The electrical resistivities evaluated by using PDs at points A and B of Figs. 3-5 are listed in Table 1. The values of the potential drops at the first acquisition for oxidized samples in all the three cases are very close to one another. Therefore, the obtained values $\rho_{1}$ of the oxide scale are almost same although the decreasing nature of potential drop differs in the three cases. This nature has no effect on the evaluation of electrical resistivity of oxide scale as the first acquisition of PD in each case was used in the evaluation. The thickness of the oxide scale in any case is so small compared to the probe spacing that it can be neglected, in evaluation of the electrical resistivity, which is the condition of derivation of Eq. (2). Therefore, the evaluation of the electrical resistivity of the oxide layer will not be influenced by the thickness of the oxide layer.

The resistivity of the oxide layer is found to be slightly varied in the range $5.64 \times 10^{-4}$ to $7.68 \times 10^{-4} \Omega \cdot \mathrm{m}$ as shown in Table 1 . These values are very high compared to the resistivity of base material $\left(20.0 \times 10^{-8} \Omega \cdot \mathrm{m}\right)$. This large difference in resistivity of two layers makes the method easy for determining their resistivities sensitively and accurately. The oxide material formed on carbon steel at the temperature $T \leq 533 \mathrm{~K}$ is thermodynamically stable

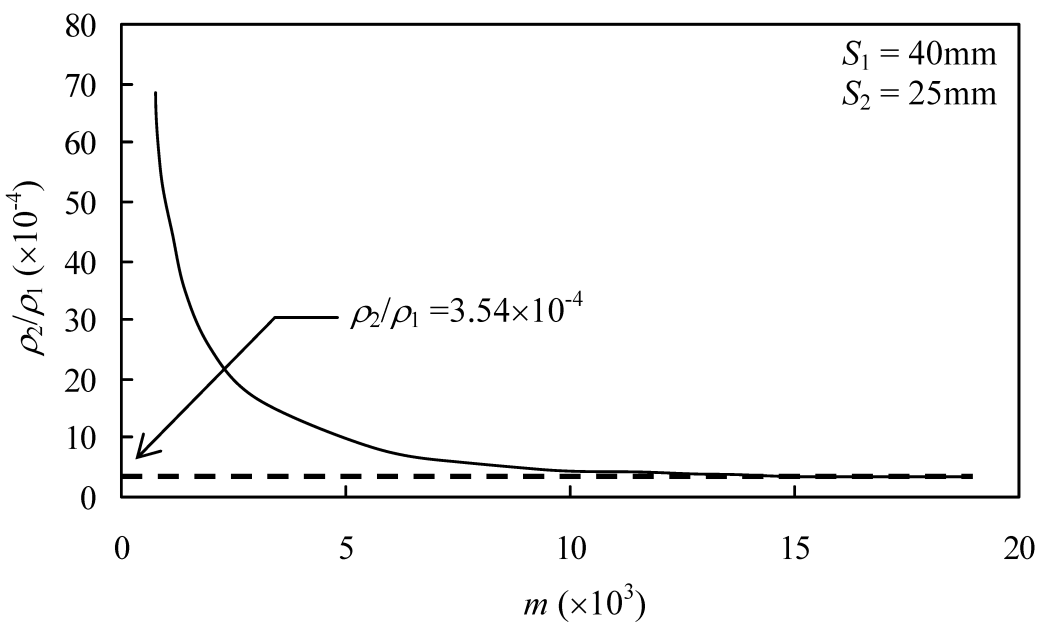

Fig. 7 Graphical result of Eq. (2) for short exposure carbon steel sample (S-1) in atmosphere.

magnetite $\left(\mathrm{Fe}_{3} \mathrm{O}_{4}\right)^{(16)}$. In our case, the three samples were prepared within the temperature range from room condition to $324.6 \mathrm{~K}$. Therefore, the oxide materials of the three samples are expected be magnetite and their electrical resistivities should be unique. The evaluated electrical resistivity of oxide scale $\rho_{1}$ as shown in Table 1 has good agreement with reported electrical resistivity range $1.00 \times 10^{-4} \sim 6.90 \times 10^{-4} \Omega \cdot \mathrm{m}$ of magnetite oxide scale ${ }^{(17-21)}$. This indicates that the oxide materials are similar in type in respect to electrical resistivity even though scale materials are formed in three different conditions. 
Table 1 Electrical resistivities of oxide layer and base material.

\begin{tabular}{|c|c|c|c|c|}
\hline Sample & Sample conditions & $\rho_{2}(\Omega \cdot \mathrm{m})$ & $\rho_{2} / \rho_{1}$ & $\rho_{1}(\Omega \cdot \mathrm{m})$ \\
\hline \multirow[t]{2}{*}{ S-1 } & Short exposure period & & $\begin{array}{c}3.54 \times 10^{-4} \\
\text { (from Fig. } 7 \text { ) }\end{array}$ & $5.64 \times 10^{-4}$ \\
\hline & After rubbing & $20.0 \times 10^{-8}$ & & \\
\hline \multirow[t]{2}{*}{ S-2 } & Long exposure period & & $2.92 \times 10^{-4}$ & $6.84 \times 10^{-4}$ \\
\hline & After rubbing & $20.0 \times 10^{-8}$ & & \\
\hline \multirow[t]{2}{*}{ S-3 } & Heat treated & & $2.60 \times 10^{-4}$ & $7.68 \times 10^{-4}$ \\
\hline & After rubbing & $20.0 \times 10^{-8}$ & & \\
\hline
\end{tabular}

\section{Conclusions}

Potential drop data for the oxide scale free surface of carbon steel has no variation with acquisition time, whereas these data for the oxidized surface show decreasing nature from a higher value to the value very close to the potential drop for the base material with the acquisition time. Therefore, the condition of surface (oxidized or not) can be easily understood from the nature of potential drop measurements. The electrical resistivities of the scale materials of the samples are successfully evaluated. Potential drops at the first acquisition for the oxidized surface in all the three cases are found very close to one another which give the similar magnitude of electrical resistivities of the oxide scales. Therefore, the oxide scales formed in the three different conditions are the same type in respect to electrical resistivity. From the behavior of potential drop decreasing nature against acquisition time, thickness and/or mechanical properties of the oxide scale deposited on the sample subjected to heat treatment at low temperature might be different than that of the sample experienced short exposure. Potential drop decreasing nature with acquisition time for the oxidized test samples depends on the thickness and/or mechanical properties of the oxide scale. However the difference in decreasing nature of potential drop has no influence on the evaluation of electrical resistivity as the first acquisition of potential drop was only used for evaluation of electrical resistivity.

\section{Acknowledgement}

The first author is pleased to acknowledge the financial support through scholarship program by the Japanese Ministry of Education, Culture, Sports, Science, and Technology (MEXT).

\section{References}

(1) Johnson, E. W. and Johnson, H. H., Technique for Measuring Position Dependent Electrical Resistivity, The Review of Scientific Instruments, Vol.35, No.11(1964), pp.1510-1516.

(2) Nishino, Y., Electrical Resistance Anomaly in $\mathrm{Fe}_{3} \mathrm{Al}$-based Alloys, Materials Science and Engineering: A, Vol.258, No.1(1998), pp.50-58.

(3) MacDonald, D. K. C., Thermoelectricity: An Introduction to the Principles, (1962), pp.1-2, John Wiley \& Sons.

(4) Wielant, J., Posner, R., Grundmeier, G. and Terryn, H., Interface Dipoles Observed after Adsorption of Model Compounds on Iron Oxide Films: Effect of Organic Functionality and Oxide Surface Chemistry, The Journal of Physical Chemistry C, Vol.112, No.33(2008), pp.12951-12957.

(5) Wielant, J., Goossens, V., Hausbrand, R. and Terryn, H., Electronic Properties of 
Thermally Formed Thin Iron Oxide Films, Electrochimica Acta, Vol.52, No.27(2007), pp.7617-7625.

(6) Jie, S., De-ren, W., Ye-dong, H., Hui-bin, Q. and Gao, W., Reduction of Oxide Scale on Hot-rolled Strip Steels by Carbon Monoxide, Materials Letters, Vol.62, No.20(2008), pp.3500-3502.

(7) Sun, W., Tieu, A. K., Jiang, Z. and Lu, C., High Temperature Oxide Scale Characteristics of Low Carbon Steel in Hot Rolling, Journal of Materials Processing Technology, Vol.155-156(2004), pp.1307-1312.

(8) Colas, R., Modelling Heat Transfer During Hot Rolling of Steel Strip, Modelling and Simulation in Materials Science and Engineering, Vol.3, No.4(1995), pp.437-453.

(9) Milan, J. C. G., Carvalho, M. A., Xavier, R. R., Franco, S. D. and De Mello, J. D. B., Effect of Temperature, Normal Load and Pre-oxidation on the Sliding Wear of Multi-component Ferrous Alloys, Wear, Vol.259, No.1-6(2005), pp.412-423.

(10) Mes'kin, V. S., Mishkevich, R. I., Gauptman, A. G. and Korolev, N. V., Magnetic Properties of Cold-rolled Electrical Steel after Hot-air Oxidation, Metal Science and Heat Treatment, Vol.9, No.2(1967), pp.145-148.

(11) Ali, M. R., Saka, M. and Tohmyoh, H., Checking Surface Contamination and Determination of Electrical Resistivity of Oxide Scale Deposited on Low Carbon Steel by DC Potential Drop Method, Materials Transactions, Vol.51, No.8(2010), pp.1414-1419.

(12) Ahmed, S. R. and Saka, M., Enhancing Repeatability in the Measurement of Potential Drop using an Adjustable Four-point-probe Measuring System, Research in Nondestructive Evaluation, Vol.18, No.2(2007), pp.69-100.

(13) Telford, W. M., Geldart, L. P., Sheriff, R. E. and Keys, D. A., Applied Geophysics, (1976), pp.643-647,Cambridge University Press.

(14) Takeo, F., Saka, M. and Sagae, T., Distinction of Alloy Steels by Means of the Closely Coupled Probes Potential Drop Technique, Proceedings of 15th World Conference on Nondestructive Testing, (2000), pp.1-5.

(15) Konchits, V. V. and Kim, C. K., Electric Current Passage and Interface Heating, Wear, Vol.232, No.1(1999), pp.31-40.

(16) Henshall, G. A., Numerical Predictions of Dry Oxidation of Iron and Low-carbon Steel at Moderately Elevated Temperatures, MRS Symposium Proceedings, (1996), pp. 667-673.

(17) Coey, J. M. D., Berkowitz, A. E., Balcells, L., Putris, F. F. and Parker, F. T., Magnetoresistance of Magnetite, Applied Physics Letters, Vol.72, No.6(1998), pp.734-736.

(18) Hsu, J. H., Chen, S. Y. and Chang, C. R., Anomalous Positive Magnetoresistance in $\mathrm{Fe}_{3} \mathrm{O}_{4}$-Ag Composite Films, Journal of Magnetism and Magnetic Materials, Vol.242-245,Part 1(2002), pp.479-481.

(19) Maris, G., Shklyarevskii, O., Jdira, L., Hermsen, J. G. H. and Speller, S., One-dimensional Structural and Electronic Properties of Magnetic $\mathrm{Fe}_{3} \mathrm{O}_{4}(110)$, Surface Science, Vol.600, No.23(2006), pp.5084-5091.

(20) Liu, H., Jiang, E. Y., Bai, H. L., Zheng, R. K. and Zhang, X. X., Thickness Dependence of Magnetic and Magneto-transport Properties of Polycrystalline $\mathrm{Fe}_{3} \mathrm{O}_{4}$ Films Prepared by Reactive Sputtering at Room Temperature, Journal of Physics D: Applied Physics, Vol.36, No.23(2003), pp.2950-2953.

(21) Peng, Y., Park, C. and Laughlin, D. E., $\mathrm{Fe}_{3} \mathrm{O}_{4}$ Thin Films Sputter Deposited from Iron Oxide Targets, Journal of Applied Physics, Vol.93, No.10 (2003), pp.7957-7959. 